EESTI NSV TEADUSTE AKADEEMIA TOIMETISED. XIV KÖIDE FOOSIKA-MATEMAATIKA- JA TEHNIKATEADUSTE SEERIA. 1965, NR. 3

ИЗВЕСТИЯ АКАДЕМИИ НАУК ЭСТОНСКОИ ССР. ТОМ ХІV СЕРИЯ ФИЗИКО-МАТЕМАТИЧЕСКИХ И ТЕХНИЧЕСКИХ НАУК. 1965, № 3

\title{
ПРОГРАММА МЕТОДА ТРЕХМЕРНЫХ СЕТОК ДЛЯ АНАЛИЗА ПЕРЕХОДНОГО ВОЛНОВОГО ПРОЦЕССА ДЕФОРМАЦИИ ПЛИТЫ
}

При импульсных нагрузках в технических приложениях представляет интерес изучение начала движения плиты на основе уравнений теории упругости. Поскольку эти уравнения не поддаются аналитическому решению, заслуживает внимания использование конечно-разностных методов. В связи с этим в работе [ $\left.{ }^{1}\right]$, помещенной в настояцем выпуске, предлагается алгоритм метода трехмерных сеток и приводятся некоторые результаты его применения. Данная заметка затрагивает вопросы реализации этого алгоритма на электронной вычислительной машине (ЭВМ).

Задача сформулирована в работе [']. Рассматривается переходный волновой процесс изгиба плиты, зависящий от безразмерной (деленной на полутолщину плиты $h$ ), продольной координаты $\xi$, безразмерной (деленной на $h$ ) нормальной координаты и безразмерного времени $\tau=c_{2} t h^{-1}$ (здесь $t-$ время, $c_{2}-$ скорость распространения волн сдвига). Предполагается, что волновой процесс возбужден импульсной нагрузкой, действующей в поперечном сечении $\xi=0$. Поскольку напряженное состояние изгиба является антисимметричным относительно срединной поверхности плиты $\zeta=0$, ограничимся рассмотрением верхней половины плиты. Итак, область изменения $\xi$,,$\tau$ ограничена условиями

$$
\xi \geqslant 0, \quad 0 \leqslant \xi \leqslant 1, \quad \tau \geqslant 0 .
$$

Расчет ведется по слоям $\tau=$ const, причем на каждом слое учитываются краевые условия при $\xi=0$, фронтовые условия при $\xi=\tau k^{-1}$, условия антисимметрии при $\zeta=0$, условня отсутствия нормальных напряжений $\left(\sigma_{33}\right)$ и тангенциальных напряжений $\left(\sigma_{13}\right)$ при $\zeta=1$ (здесь $k=c_{2} / c_{1}-$ соотношение скоростей распространения волны сдвига и сжатия). Расчетные формулы для перемещений $u$, w даны там же [']. При их программнровании пришлось преодолеть следующие затруднения:

1. Условия $\sigma_{33}=\sigma_{13}=0$ в точках сетки, где $\zeta=1$, образуют систему уравнений, причем число уравнений растет с увеличением $\tau$ и достигает в конкретных задачах весьма больших значений. В последнем слое, вычисленном для примера работы [1], оно дошло до 504. Чтобы избежать итеративного решения таких больших систем уравнений и повысить точность расчета, решение было построено в виде рекуррентных формул.

2. Объем оперативной памяти ЭВМ составляет обычно 2048 или 4096 слов. Однако при решении прикладных задач методом трехмерных сеток оказывается необходимым сохранить больше информации (например, на последнем этапе решения рассмотренной задачи ['] - около 30000 слов). Поэтому слои $\tau=$ const были разбиты на области (см. рис. 1) так, чтобы в пределах каждой области расчет можно было провести с применением только оперативной памяти. При переходе от одной области к другой нмеет место обмен информации между оперативной и внешней памятью.

\section{1. Схема расчета.}

В областях $m=2,3, \ldots$, M-2 расчет проводится одинаково (см. блок-схему I, рис. 2); область $m=1$ отличается тем, что при $\xi=0$ учитываются краевые условия; область $m=M-1 \quad$ отличается

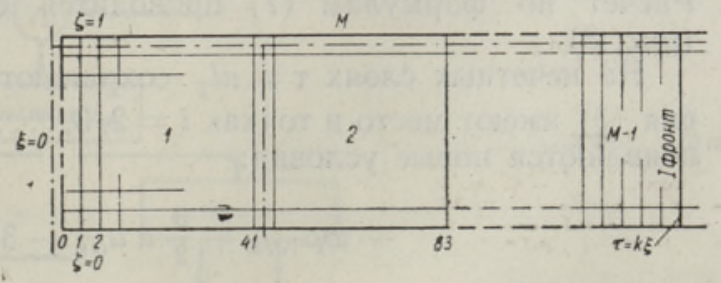

Рис. 1.

тем, что в прифронтовых точках применяются основные и дополнительные фронтовые условия. Расчетные формулы даны в [1]. 
О бла сть $m=M$. На четных слоях $\tau=n l_{\tau}$ имеем следующие условия (см. формулы $(1.24)-(1.26)$ работы [1]):

при $i=0: \quad w_{0 J n}=0, u_{0 J n}=-\frac{2}{3} A l_{\xi}+\frac{4}{3} u_{1 J n}-\frac{1}{3} u_{2 J n}$

при $i=1: \quad a u_{1 J n}+w_{2 J n}=A_{12}$

$$
\left.\begin{array}{l}
a u_{1 J n}+w_{2 J n}=A_{12} \\
-\frac{4}{3} u_{1 J n}+b w_{1 J n}+\frac{4}{3} u_{2 J n}=A_{11}
\end{array}\right\}
$$

при $\left.i=2,3, \ldots, \begin{array}{rl}\frac{n}{2}-1: & -w_{i-1, \text { In }}+a u_{i J n}+w_{i+1, J n}=A_{i 2} \\ & -u_{i-1, J n}+b w_{i J n}+u_{i+1, J n}=A_{i 1}\end{array}\right\}$

при $i=\frac{n}{2}: \quad u_{1 J n}=0, w_{i J n}=0$,

где $A_{11}=\frac{b}{3}\left(4 w_{1, J-1, n}-w_{1, J-2, n}\right)-\frac{2(1-2 v)}{3(1-v)^{2}} l_{\xi}$,

$$
\begin{array}{cc}
A_{i 1}=\frac{b}{3}\left(4 w_{i, J-1, n}-w_{i, J-2, n}\right), & \text { если } i \geqslant 2 \\
A_{i 2}=\frac{a}{3}\left(4 u_{i, J-1, n}-u_{i, J-2, n}\right), & \text { если } i \geqslant 1 \\
a=3 \frac{l_{\xi}}{l_{\zeta}}, \quad b=3 \frac{1-v}{v} \frac{l_{\xi}}{l_{\zeta}} . &
\end{array}
$$

Условия (2) и (3) образуют систему из $n-2$ уравнений. Преобразованием матрицы к нижнетреугольной форме было получено решение в виде следующих рекуррентных формул:

$$
\begin{gathered}
u_{i J n}=B_{i 2}+r_{i 2} w_{i-1, J_{n}}, \quad w_{i J n}=B_{i 1}+r_{i 1} u_{i-1, J n} \\
w_{0 J n}=0, \quad w_{1 J n}=B_{11}+\frac{4}{3} r_{11} u_{1 J n},
\end{gathered}
$$

где использовались обозначения

$$
\begin{aligned}
& B_{i 2}=r_{i 2}\left(A_{i 2}-B_{i+1,1}\right), \quad B_{i 1}=r_{i 1}\left(A_{i 1}-B_{i+1,2}\right) \\
& B_{11}=r_{11}\left(A_{11}-\frac{4}{3} B_{22}\right), \quad B_{\frac{n}{2}, 2}=B_{\frac{n}{2}, 1}=0 \\
& r_{2}=\frac{1}{a+r_{i+1,1}}, \quad r_{i 1}=\frac{1}{b+r_{i+1,2}}, \quad r_{11}=\frac{1}{b+\frac{4}{3} r_{22}}, \quad r_{\frac{n}{2}, 2}=r_{\frac{n}{2}, 1}=0 .
\end{aligned}
$$

Расчет по формулам (7) проводится с применением блок-схемы II (рис. 3).

На нечетных слоях $\tau=n l_{\tau}$ сохраняют силу условия (1) и (2); условия (3) имеют место в точках $i=2,3, \ldots, \frac{1}{2}(n-3)$; в точке $i=\frac{1}{2}(n-1)$ появляются новые условия:

$$
\begin{aligned}
& -w_{i-1, J n}+\frac{3}{2} a u_{i J n}-3 w_{i J n}=\frac{3}{2} A_{i 2} \\
& -u_{i-1, J n}+\frac{3}{2} b w_{i J n}-3 u_{i J n}=\frac{3}{2} A_{i 1},
\end{aligned}
$$

а при $i=\frac{1}{2}(n+1)$ имеют место условия (4). 
Условия (2), (3) и (8) образуют систему из $n-1$ уравнений. При построении решения в виде рекуррентных формул оказалось (в отличие от предыдущего случая) целесообразным привести матрицу к верхнетреугольной форме. Были получены следующие расчетные формулы:

$$
\left.\begin{array}{l}
u_{i J n}=B_{i 2}-r_{i 2} w_{i+1, J n}-C_{i 2} \\
w_{i J n}=B_{i 1}-r_{i 1} u_{i+1, J n}-C_{i 1}
\end{array}\right\} \quad i \leqslant \frac{n-3}{2}
$$

$$
u_{i J n}=B_{i 2}+3 r_{i 2} w_{i J n}-C_{i 2}, \quad u_{i+1, J n}=0, \quad w_{i+1, J_{n}}=0, \quad i=\frac{1}{2}(n-1),
$$

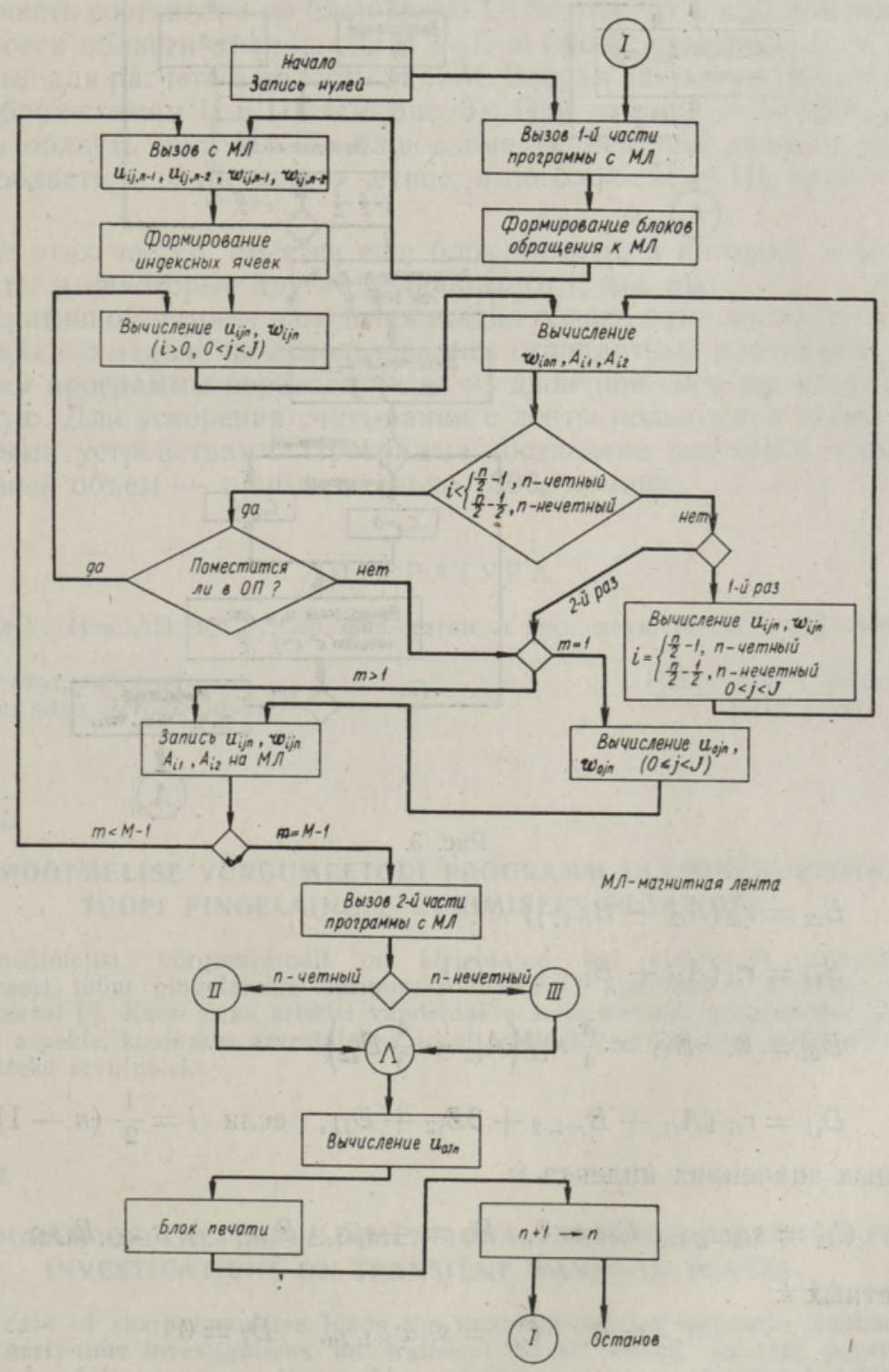

Рис. 2. 

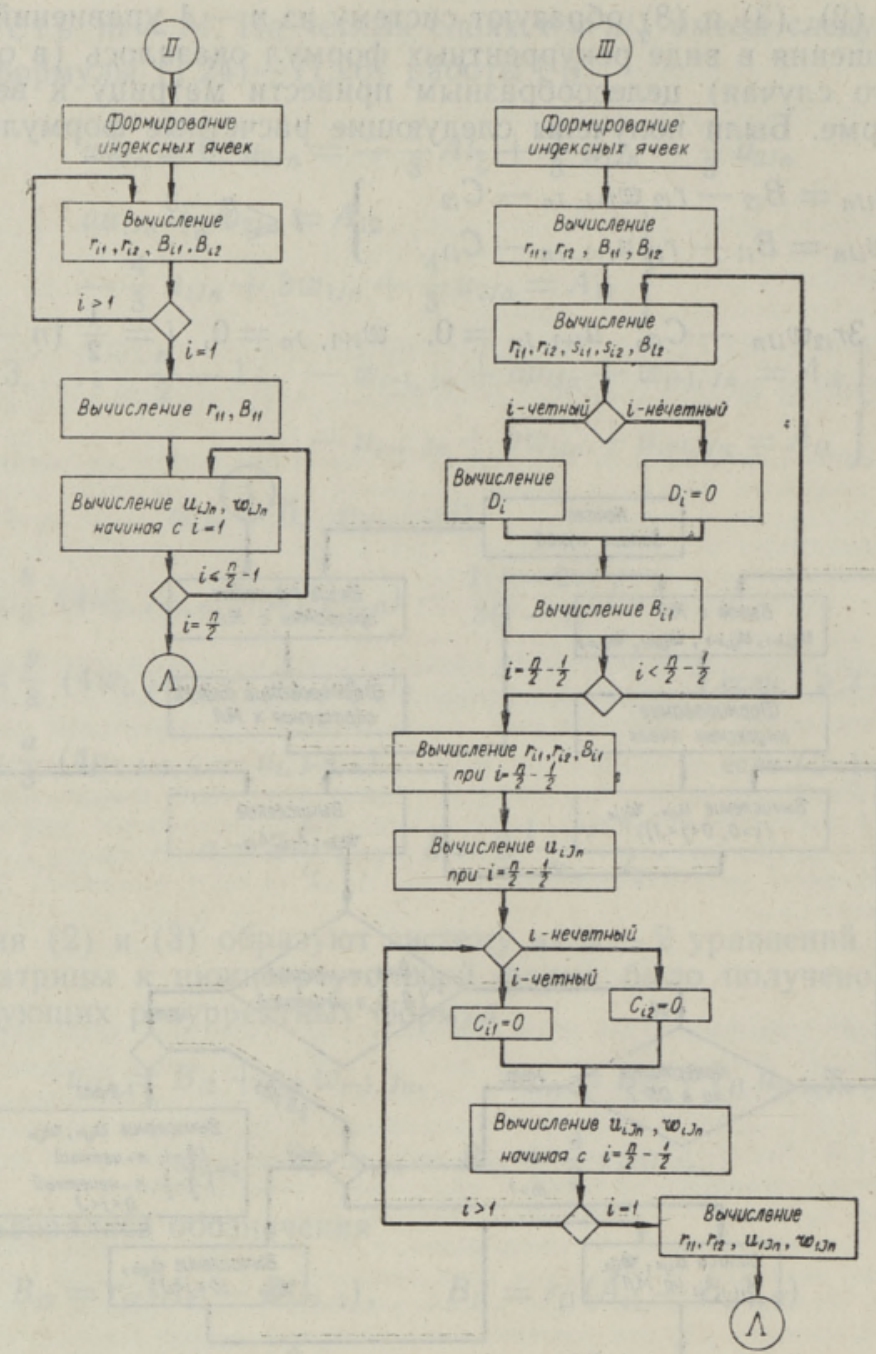

Рис. 3.

$$
\begin{aligned}
& B_{i 2}=r_{i 2}\left(A_{i 2}+B_{i-1,1}\right) \\
& B_{i 1}=r_{i 1}\left(A_{i 1}+B_{i-1,2}+D_{i}\right) \\
& B_{01}=0, \quad B_{11}=\frac{3}{4} r_{11}\left(A_{11}+\frac{4}{3} B_{12}\right) \\
& B_{i 1}=r_{i 1}\left(A_{i 1}+B_{i-1,2}+3 B_{i 2}+D_{i}\right), \quad \text { если } i=\frac{1}{2}(n-1) ;
\end{aligned}
$$

при четных значениях индекса $i$ :

$$
C_{i 2}=s_{i 2} w_{i J n}, \quad C_{i 1}=0, \quad D_{i}=-s_{i-1,2} B_{i-1,1}+s_{i-1,1} B_{i, 2} ;
$$

при нечетных $i$ :

$$
\begin{gathered}
C_{i 2}=0, \quad C_{i 1}=s_{i 1} w_{i+1, J n}, \quad D_{i}=0 \\
r_{i 2}=\frac{1}{a+r_{i-1,1}}, \quad r_{i 1}=\frac{1}{b+r_{i-1,2}}, \quad r_{12}=\frac{1}{a}, \quad r_{11}=\frac{4}{3 b}
\end{gathered}
$$




$$
\left.\begin{array}{c}
r_{i 2}=\frac{1}{\frac{3}{2} a+r_{i-1,1}} \\
r_{i 1}=\frac{1}{\frac{3}{2} a+r_{i-1,2}+3 r_{i 2}\left[-3+(-1)^{i} s_{i-1,1}\right]}
\end{array}\right\} i=\frac{1}{2}(n-1)
$$

Расчет по формулам (9) проводится с применением блок-схемы III.

2. Блок-схема программы. Программа состой из двух частей. Первая часть составлена по блок-схеме I (см. рис. 2) и с ее помощью рассчитываются области $m=1, \ldots, M-1$; а также величины $A_{i 1}$ и $A_{i 2}$, необходимые для расчета в области $m=M$. Вторая часть программы составлена по блок-схемам II и III (см. рис. 3). При помощи этой части рассчитывается область $m=M$. По блок-схеме II ведутся расчеты на слое $\tau=n l_{\tau}$ области $m=M$, если $n$ четное, и по блок-схеме III, если $n-$ нечетное.

Кроме этих частей имеется еще блок печати, в котором печатаются результаты и некоторые другие величины $\left(\sigma_{11}, \sigma_{13}, \sigma_{33}\right)$.

В оперативной памяти находится всегда только одна часть программы.

При данной задаче не использовались стандартные программы, за исключением программы перевода чисел из двоичной системы исчисления в десятичную. Для ускорения считывания с ленты пользуются тремя лентопротяжными устройствами. Программа составлена для ЭВМ «Минск-2» и ее полный объем - приблизительно $1300_{8}$ команд.

ЛИТЕРАТ У РА

1. Н и гу л У., Изв. АН ЭССР. Сер. физ.-матем. и техн. наук, 14, № 3, 345-384 (1965).

Ннститут кибернетики

Академии наук Эстонской ССР
Поступила в редакцию 16/IV 1965

\section{A. MÄNNIL}

\section{KOLMEMÕTTMELISE VÕRGUMEETODI PROGRAMM OLEMINEKUPROTSESSI TUOPI PINGELAINETE UURIMISEKS PLAATIDES}

Kolmemõōtmelist võrgumeetodit on kirjeldatud kui efektiivset vahendit üleminekuprotsessi tüüpi pingelainete uurimiseks lühikestel ajalōikudel järskude impulsskoormiste korral [']. Käesolevas artiklis vaadeldakse selle meetodi programmeerimisprobleemi üldisi aspekte, kusjuures arvestatakse arvuti «Minsk-2» võimalusi, mida autor kasutas praktilisteks arvutusteks.

\section{A. $M \ddot{A N N I L}$}

\section{A PROGRAM OF THE THREE-DIMENSIONAL NETWORKS METHOD FOR INVESTIGATIONS ON TRANSIENT WAVES IN PLATES}

In the case of sharp impulsive loads the three-dimensional networks method $\left.{ }^{1}{ }^{1}\right]$ is efficient in early-time investigations on transient stress waves. In this paper some general aspects of the programming problem of this method are discussed, taking into consideration the possibilities of the computer "Minsk-2" used by the author in the practical computations. 